\title{
Some Questions of Number-theoretical Method in Approximation Analysis
}

\section{P. Dobrovolskaya ${ }^{1}$, M. N. Dobrovolsky ${ }^{2}$, N. M. Dobrovol'skii ${ }^{3}$, N. N. Dobrovol'skii ${ }^{4}$, I. Y. Rebrova ${ }^{5}$}

\footnotetext{
${ }^{1}$ Institute of Economics and Management, Russia, 300041, Tula, Veresaeva st., Ibocharova6565@mail.ru

${ }^{2}$ Geophysical center RAS, Russia, 119296, Moscow, Molodezhnaya str., 3, dobrovolsky.michael@gmail.com

${ }^{3}$ Tula State Pedagogical University, Russia, 300026, Tula, pr. Lenina, 125, dobrovol@ tspu.tula.ru

${ }^{4}$ Tula State University, Russia, 300600, Tula, pr. Lenina, 92, nikolai.dobrovolsky@gmail.com

${ }^{5}$ Tula State Pedagogical University, Russia, 300026, Tula, pr. Lenina, 125, i_rebrova@mail.ru
}

This article gives an overview of several actual problems of optimal coefficients method. This overview was done on September 12,

2013 on XI internation conference «Algebra and number theory: modern problems and applications» in Saratov city.

Key words: optimal coefficients method, algebraic lattices, Gelfond theorem, hyperbolic zeta-function.

\section{References}

1. Korobov N. M. Teoretiko-chislovye metody v priblizhennom analize [Number-theoretic methods in approximations analysis]. Moscow, 2004 (in Russian).

2. Dobrovolskiy N. M. Giperbolicheskaia dzeta-funktsiia reshetok [Hyperbolic zeta-function on lattices]. Tula, 1984. Dep. v VINITI 24.08.84, no. 6090-84 (in Russian). 3. Dobrovolskaya L. P., Dobrovolskiy M. N., Dobrovolskiy N. M., Dobrovolskiy N. N. Giperbolicheskie dzetafunktsii setok $i$ reshetok $i$ vychislenie optimal'nykh koeffitsientov [Hyperbolic zeta-functions on nets and lattices and computation of optimal coefficients]. Chebyshevskii sbornik [Chebyshev collection], 2012, vol. 13, iss. 4(44), pp. 4-107 (in Russian).

4. Dobrovolskaya L. P., Dobrovolskiy M. N., Dobrovolskiy N. M., Dobrovolskiy N. N. Mnogomernye teoretiko- chislovye setki $i$ reshetki $i$ algoritmy poiska optimal'nykh koeffitsientov [Multidimensional number-theoretic nets and lattices and their applications]. Tula, State Pedagogic University Press, 2005, 195 p. (in Russian).

5. Dobrovolskiy N. M. Mnogomernye teoretiko-chislovye setki $i$ reshetki $i$ ikh prilozheniia [Multidimensional number-theoretic nets and lattices and their applications]. Tula, State Pedagogic University Press, 2005, 195 p. (in Russian).

6. Dobrovolskiy M. N. Funktsional'noe uravnenie dlia giperbolicheskoi dzeta-funktsii tselochislennykh reshetok [Functional equation of hyperbolic zeta-function on integral lattices]. Vestn. Mosk. un-ta. Ser. 1. Matematika. Mekhanika, 2007, iss. 3, pp. 18-23 (in Russian).

УДК 512.567.5

\section{ОБ УСЛОВИЯХ ДИСТРИБУТИВНОСТИ И МОДУЛЯРНОСТИ РЕШЕТОК КОНГРУЭНЦИЙ КОММУТАТИВНЫХ УНАРНЫХ АЛГЕБР}

\author{
В. К. Карташов ${ }^{1}$, А. В. Карташова ${ }^{2}$, В. Н. Пономарёв
}

\begin{abstract}
${ }^{1}$ Кандидат фризико-математических наук, заведующий кафредрой алгебры, геометрии и математического анализа, Волгоградский государственный социально-педагогический университет, kartashovvk@yandex.ru

${ }^{2}$ Кандидат фризико-математических наук, доцент кафредры алгебры, геометрии и математического анализа, Волгоградский государственный социально-педагогический университет, kartashovaan@yandex.ru

Статья посвящена известной проблеме описания унарных алгебр, решетки конгруэнций которых обладают заданным свойством. К настоящему времени эта проблема решена для унарных алгебр с одной операцией. Показано, что дляпроизвольных коммутативных унарных алгебр данная проблема является гораздо более сложной. Здесь приводится несколько необходимых условий дистрибутивности и модулярности таких решеток. Доказано также, что решетка всех подмножеств любого множества изоморфрна решетке конгруэнций подходящей связной коммутативной унарной алгебры.
\end{abstract}

Ключевые слова: коммутативная унарная алгебра, дистрибутивная решетка, модулярная решетка, решетка конгруэнций алгебры. 


\section{ВВЕДЕНИЕ}

Как известно (см., например, [1]), решетка Con $A$ конгруэнций произвольной алгебры $\mathfrak{A}$ содержит значительную информацию о свойствах самой алгебры $\mathfrak{A}$. Поэтому проблема описания класса алгебр фиксированной сигнатуры, решетки конгруэнций которых обладают заданным свойством, привлекает внимание многих математиков.

Вопросы, связанные с исследованием решеток конгруэнций алгебр, занимают значительное место в работах Г. Гретцера (G. Gratzer), А. И. Мальцева, Л. А. Скорнякова и других алгебраистов (см., например, [1-9]).

В работе Г. Гретцера и Е. Т. Шмидта (Е. T. Shmidt) [1] эта проблема была сведена к исследованию решеток конгруэнций унарных алгебр. Ими было доказано, что для любой алгебры $\mathfrak{A}$ существует унарная алгебра $\mathfrak{B}$ такая, что $\operatorname{Con} \mathfrak{A} \cong C \operatorname{Con} \mathfrak{B}$. Это повысило интерес к данной проблеме, а также к другим вопросам, связанным с унарными алгебрами.

Унарные алгебры имеют глубокие связи с другими разделами универсальной алгебры. В частности, любая унарная алгебра может быть интерпретирована как автомат без выхода, как полигон либо как ориентированный граф.

Кроме того, в отличие от классических алгебр (групп, колец, полугрупп и т. д.) унарные алгебры имеют ряд особых свойств. Поэтому исследование их весьма важно для построения общей теории алгебраических систем.

$\mathrm{K}$ настоящему времени по указанной выше проблеме для класса унаров (алгебр с одной унарной операцией) существует ряд глубоких результатов, имеющих окончательный характер. В частности, полностью описаны классы унаров, решетка конгруэнций которых модулярна, дистрибутивна, стоунова, булева, с дополнениями, а также - решены многие другие классические вопросы,которые возникают для решеток, связаенных с каким-нибудь фиксированным классом алгебр [4-8].

Для унарных алгебр, сигнатура которых содержит более одной операции, эти задачи оказались значительно более сложными.

Например, известно [7], что любой однопорожденный унар имеет дистрибутивную решетку конгруэнций. Однако в общей ситуации это утверждения неверно.

Для сравнения приведем следующие два примера.

Пример 1. $\mathfrak{A}_{1}=\langle A, f, g\rangle$, где $A=\{a, b, c, d\}, f(a)=b, f(b)=a, f(c)=d, f(d)=c$ и $g(a)=g(c)=c, g(b)=g(d)=d$.

Пример 2. $\mathfrak{A}_{2}=\langle A, f, g\rangle$, где $A=\{a, b, c, d\}, f(a)=b, f(b)=a, f(c)=d, f(d)=c$ и $g(a)=c$, $g(b)=d, g(c)=a, g(d)=b$.

Первая алгебра порождается любым из элементов $a, b$, вторая - любым ее элементом. Непосредственная проверка показывает, что решетки конгруэнций обеих алгебр пятиэлементны, при этом Con $\mathfrak{A}_{1}$ немодулярна, а Сon $\mathfrak{A}_{2}$ модулярна, но не дистрибутивна.

В данной статье приводится несколько необходимых условий модулярности и дистрибутивности коммутативных унарных алгебр.

Напомним, что унарная алгебра $\langle A, \Omega\rangle$ называется коммутативной, если $f g(x)=g f(x)$ для любых $f, g \in \Omega$ и $x \in A$.

Очевидно, что алгебры, указанные в примерах 1 и 2 , являются коммутативными.

\section{1. ОСНОВНЫЕ ОПРЕДЕЛЕНИЯ И ОБОЗНАЧЕНИЯ}

Далее, $\mathbb{N}$ означает множество положительных целых чисел и $\mathbb{N}_{0}=\mathbb{N} \cup\{0\}$.

Пусть $\mathfrak{A}=\langle A, \Omega\rangle$ - произвольная унарная алгебра. Через $\Omega^{*}$ обозначается свободный моноид слов с порождающим множеством $\Omega$ относительно композиции. Единицей в $\Omega^{*}$ служит пустое слово $\emptyset$.

Результат $w(a)$ применения слова $w \in \Omega^{*}$ к элементу $a \in A$ определяется индуктивно по длине 
слова (см., например, [2]). По определению полагаем $f^{0}(a)=\emptyset a=a, f^{n}(a)=f\left(f^{n-1}(a)\right)$ для произвольных $f \in \Omega, a \in A$ и $n \in \mathbb{N}$.

Отсюда, если $w=w_{1} w_{2}$, то $w(a)=w_{1}\left(w_{2}(a)\right)$, где $w, w_{1}, w_{2} \in \Omega^{*}$ и $a \in A$.

Далее, для любого элемента $a \in A$ через $(a)$ обозначается подалгебра алгебры $\mathfrak{A}$, порожденная элементом $a$.

Для любой подалгебры $\mathfrak{B}=\langle B, \Omega\rangle$ алгебры $\mathfrak{A}=\langle A, \Omega\rangle$ бинарное отношение $\rho_{B}$ на множестве $A$, определенное по правилу

$$
x \rho_{B} y \Leftrightarrow(x=y) \vee\{x, y\} \subseteq B,
$$

очевидно является конгруэнцией алгебры $\mathfrak{A}$. Эта конгруэнция называется конгруэнщией Pucca, соответствующей подалгебре $\mathfrak{B}$.

Алгебра называется связной, если $(a) \cap(b) \neq \emptyset$ для любых ее элементов $a$ и $b$, и сильно связной, если она порождается любым своим элементом.

Элементы $a, b \in A$ называются взаимно достижимыми, если $(a)=(b)$.

Очевидно, что отношение взаимной достижимости является эквивалентностью на множестве $A$. В дальнейшем ради краткости в случае, если элементы $a$ и $b$ находятся в этом отношении, будем говорить, что элементы $a$ и $b$ эквивалентны и писать $a \sim b$.

Лемма 1 [9, лемма 3]. Отношение эквивалентности между элементами сохраняется при гомоморфизме унарных алгебр, то есть если $\varphi: A \rightarrow B-$ некоторый гомоморфизм унарных алгебр $\mathfrak{A} u \mathfrak{B}, a, b \in A$ u $a \sim b, \operatorname{mo} \varphi(a) \sim \varphi(b)$.

Класс эквивалентности $\sim$ с порождающим элементом $a$ называется слоем этого элемента и обозначается через $S(a)$.

Множество $\Omega(a)=\{f \in \Omega \mid f(a) \sim a\}$ называется сигнатурой слоя $S(a)$.

Заметим, что $\Omega(a)$ может быть пустым. Нетрудно показать, что в случае если $\Omega(a) \neq \emptyset$, то множество $S($ а) будет сильно связной подалгеброй редукта $\langle A, \Omega(a)\rangle$.

\section{2. ОСНОВНЫЕ РЕЗУЛЬТАТЫ}

Сформулируем сначала несколько вспомогательных предложений.

Лемма 2. Пусть $\mathfrak{A}=\langle A, \Omega\rangle-$ коммутативная унарная алгебра, а $\in A$ u $\Omega(a) \neq \emptyset$. Тогда на однопорожденной подалгебре (а) истинна следующая формула:

$$
(\forall x \in(a) \forall f \in \Omega)(f(x) \in S(a) \Rightarrow x \in S(a) \& f \in \Omega(a)) .
$$

Доказательство непосредственно вытекает из определений.

Лемма 3. Если а и $b$-независимые элементы некоторой коммутативной унарной алгебры $\mathfrak{A}$, mo $S(a) \cap(b)=\emptyset$.

Доказательство также непосредственно вытекает из определений.

Лемма 4. Пусть $\mathfrak{A}=\langle A, \Omega\rangle-$ коммутативная унарная алгебра, $\left\{a_{i} \mid i \in I\right\}-$ некоторая независимая система ее порождающих, $B=A \backslash\left(\bigcup_{i \in I} S\left(a_{i}\right)\right)$. Тогда либо $B=\emptyset$, либо $\langle B, \Omega\rangle-$ подалгебра алгебры $\mathfrak{A}$.

Доказательство. Допустим, что $b \in B$ и $f \in \Omega$. Тогда $b=v\left(a_{j}\right)$ для некоторых $j \in I$ и $v \in \Omega^{*}$. Предположим, что $f(b) \notin B$. Тогда $f(b) \in S\left(a_{k}\right)$, где $k \in I$, откуда $f(b) \in\left(a_{j}\right) \cap S\left(a_{k}\right)$. Если теперь $j \neq k$, то это противоречит лемме 3 .

Пусть $j=k$. Тогда $f(b)=f\left(v\left(a_{j}\right)\right) \in S\left(a_{j}\right)$. Кроме того, $b \in\left(a_{j}\right)$. Отсюда $b \in S\left(a_{j}\right)$ в силу леммы 2 , что противоречит выбору $b$.

Пусть $A=\{a, b, c\}-$ трехэлементное множество и $\Omega=\left\{f_{1}, f_{2}, \ldots, f_{n}, \varphi_{1}, \varphi_{2}, \ldots \varphi_{k}\right\}(n, k \in \mathbb{N})-$ некоторая совокупность унарных символов. 
Построим на носителе $A$ унарную алгебру сигнатуры $\Omega$, полагая по определению:

1. $\forall x \in\{a, b\} f_{i}(x)=x(i=1,2, \ldots, n)$;

2. $\forall x \in\{a, b\} \varphi_{j}(x)=c(j=1,2, \ldots, k)$;

3. $f_{i}(c)=\varphi_{j}(c)=c(i=1,2, \ldots, n, j=1,2, \ldots, k)$.

Будем обозначать эту алгебру в дальнейшем через $\tilde{3}$.

Лемма 5. Решетка Соп $\tilde{3} \cong M_{3}$, где $M_{3}$ - пятиэлементная модулярная, но недистрибутивная решетка.

Доказательство тривиально.

Теорема 1. Если связная коммутативная унарная алгебра $\mathfrak{A}$ содержит два независимых элемента а и b таких, что сигнатуры соответствующих слоев совпадают, т. е. $\Omega(a)=\Omega(b)$, то решетка Соп недистрибутивна.

Доказательство. Пусть $\mathfrak{A}=\langle A, \Omega\rangle$ - связная коммутативная унарная алгебра, $a$ и $b$ - независимые элементы из $\mathfrak{A}$ и $\Omega(a)=\Omega(b)$.

Обозначим через $\mathfrak{B}=\langle B, \Omega\rangle$ подалгебру алгебры $\mathfrak{A}$, порожденную элементами $a$ и $b$. Так как алгебра $\mathfrak{B}$ коммутативна, то отношение эквивалентности $\sim$, определенное выше, является конгруэнцией. Из определений следует, что все слои в фактор-алгебре $\mathfrak{B} / \sim$ одноэлементны.

Очевидно также, что алгебра $\mathfrak{B}$ связна. Кроме того, как уже отмечалось выше, каждый из слоев $S(a)$ и $S(b)$ сильно связан в сигнатуре $\Omega(a)=\Omega(b)$. Отсюда в силу независимости элементов $a$ и $b$ имеем $C=B \backslash(S(a) \cup S(b)) \neq \emptyset$, откуда в силу леммы 4 получаем, что $\langle C, \Omega\rangle$ - подалгебра алгебры $\mathfrak{B}$.

Это означает, что

$$
\mathfrak{B} / \rho_{C} \cong \tilde{3},
$$

где $\rho_{C}-$ конгруэнция Рисса, соответствующая подалгебре $\langle C, \Omega\rangle$.

Теперь, учитывая, что решетка конгруэнций любой подалгебры унарной алгебры и любой ее фактор-алгебры вкладывается в решетку конгруэнций самой алгебры как подрешетка, применением леммы 5 завершаем доказательство теоремы.

Следствие. Пусть $\mathfrak{A}=\langle A, \Omega\rangle$ - связная коммутативная унарная алгебра с $n$ операциями, которая содержит независимую систему элементов $a_{1}, a_{2}, \ldots, a_{k}$, где $k \geq 2^{n}$. Тогда решетка Соп $\mathfrak{A}$ недистрибутивна.

Доказательство. Убедимся сначала, что $\Omega\left(a_{i}\right) \neq \Omega$ для всех $i \in\{1,2, \ldots, k\}$. Действительно, если $\Omega\left(a_{i}\right)=\Omega$ для некоторого $i$, то слой $\left\langle S\left(a_{i}\right), \Omega\right\rangle$ является сильно связной подалгеброй алгебры $\mathfrak{A}$. Отсюда ввиду связности алгебры $\mathfrak{A}$ для любого $j \in\{1,2, \ldots, k\}$ найдется слово $w \in \Omega^{*}$ такое, что $w\left(a_{j}\right) \in S\left(a_{i}\right)$. Это противоречит независимости системы элементов $\left\{a_{i} \mid i=1,2, \ldots, k\right\}$, поскольKy $k>1$.

Следовательно среди подмножеств $\Omega\left(a_{1}\right), \Omega\left(a_{2}\right), \ldots, \Omega\left(a_{k}\right)$ само множество $\Omega$ не встречается, откуда в силу неравенства $k \geq 2^{n}$ вытекает, что найдутся такие числа $i, j, i \neq j$, для которых $\Omega\left(a_{i}\right)=\Omega\left(a_{j}\right)$. Применяя теорему 1 , завершаем доказательство.

Следующая теорема указывает на особое место унаров в решении проблемы описания решеток конгруэнций коммутативных унарных алгебр.

Теорема 2. Пусть $\Omega$ - сигнатура, состоящая из $n$ унарных символов, где $n>1$. тогда для любого целого положительного числа $k<2^{n}$ существуют связные коммутативные унарные алгебры $\mathfrak{B}$ и $\mathfrak{C}$, у каждой из которых мощность любой независимой системь элементов не

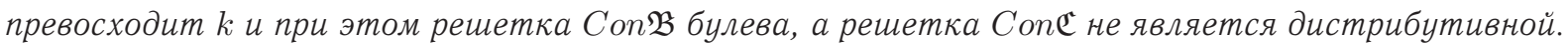

Доказательство. Пусть $n>1$ и $\Omega=\left\{f_{1}, f_{2}, \ldots, f_{n}\right\}$ - сигнатура, состоящая из $n$ унарных символов, $k \in \mathbb{N}$ и $k<2^{n}$.

I. Зафиксируем некоторую систему $\Omega_{1}, \Omega_{2}, \ldots, \Omega_{k}$ из $k$ различных подмножеств множества $\Omega$, каждое из которых отлично от $\Omega$. На носителе $B=\left\{\Omega_{1}, \Omega_{2}, \ldots, \Omega_{k}, \Omega\right\}$ зададим операции $f_{i}$ 
$(i=1,2, \ldots, n)$ по правилу $f_{i}(\Omega)=\Omega$ для всех $i \in\{1,2, \ldots, n\}$ и

$$
f_{i}\left(\Omega_{j}\right)= \begin{cases}\Omega_{j}, & \text { если } f_{i} \in \Omega_{j}, \\ \Omega, & \text { если } f_{i} \notin \Omega_{j},\end{cases}
$$

где $i \in\{1,2, \ldots, n\}, j \in\{1,2, \ldots, k\}$.

Легко проверить, что $\mathfrak{B}=\left\langle B, f_{1}, f_{2}, \ldots, f_{n}\right\rangle-$ связная коммутативная унарная алгебра, причем ее элементы $\Omega_{1}, \Omega_{2}, \ldots, \Omega_{k}$ попарно независимы.

Непосредственной проверкой убеждаемся, что любой неодноэлементный класс произвольной конгруэнции $\theta \in \operatorname{Con} \mathfrak{B}$ содержит $\Omega$. Это означает, что любая конгруэнция $\theta$ алгебры $\mathfrak{B}$ однозначно определяется классом $[\Omega]_{\theta}$. Отсюда вытекает, что

$$
\theta_{1} \subseteq \theta_{2} \Leftrightarrow[\Omega]_{\theta_{1}} \backslash\{\Omega\} \subseteq[\Omega]_{\theta_{2}} \backslash\{\Omega\}
$$

для любых конгруэнций $\theta_{1}, \theta_{2} \in \operatorname{Con} \mathfrak{B}$.

Следовательно, решетка $C$ on $\mathfrak{B}$ изоморфна решетке всех подмножеств множества $\left\{\Omega_{1}, \Omega_{2}, \ldots, \Omega_{k}\right\}$ по включению.

II. Если $k=1$, то при $n=2$ в качестве $\mathfrak{C}$ можно взять унарную алгебру, построенную в примере 1 . При $n>2$ сигнатуру этой алгебры можно дополнить, задав на ее носителе дополнительно $(n-2)$ тождественные операции.

Пусть теперь $k>1$ и $C=\left\{c_{0}, c_{1}, \ldots, c_{k}\right\}$ - некоторое множество. На множестве $C$ зададим операции $f_{i}(i=1,2, \ldots, n)$ по правилу

$$
f_{i}(x)=c_{0} \quad(i=1,2, \ldots, n)
$$

для любого $x \in C$.

Очевидно, что система элементов $c_{1}, c_{2}, \ldots, c_{k}$ независима, и $\Omega\left(c_{i}\right)=\emptyset$ для всех $i \in\{1,2, \ldots, n\}$.

Отсюда, поскольку $k>1$, получаем, что решетка Con $\mathfrak{C}$ не является дистрибутивной в силу теоремы 1.

Замечание. Теорема 2 не справедлива для унаров [7, теорема 2].

Из рассуждений, проведенных при построении алгебры $\mathfrak{B}$ в доказательстве теоремы 2, вытекает

Следствие. Решетка всех подмножеств произвольного множества изоморфна решетке конгруэнций подходящей коммутативной унарной алгебры.

Теорема 3. Если связная коммутативная унарная алгебра $\mathfrak{A}$ содержит независимое подмножество $\{a, b, c\}$ из трех элементов, у которых слои имеют одинаковую сигнатуру, то решетка Соп

Доказательство проводится по той же схеме, что и доказательство теоремы 1. На заключительном этапе получаем некоторую фактор-алгебру $\mathfrak{B}$ подалгебры алгебры $\mathfrak{A}$, порожденной множеством $\{a, b, c\}$, решетка $C$ оп $\mathfrak{B}$ конгруэнций которой изоморфна решетке всех эквивалентностей на четырехэлементном множестве.

\section{Библиографический список}

1. Gratzer G., Shmidt E. T. Characterizations of congruence lattices of abstract algebras // Acta Sci. Math. 1963. Vol. 24. P. 34-59.

2. Мальцев А. И. Алгебраические системы. М. : Наука, 1970. 392 c.

3. Skornjakov L. A. Unars // Coll. Math. Soc. J. Bolyai. 1982. Vol. 29. Universal Algebra (Esztergom 1977). P. 735-743.
4. Скорняков Л. А. Дополнения в структуре конгруенций // Мат. сб. 1972. Т. 88(130), № 5(1). С. 148-181. DOI: $10.1070 / S M 1972 v 017 n 01 \mathrm{ABEH} 001495$.

5. Berman J. On the congruence lattices of unary algebras // Proc. Amer. Math. Soc. 1972. Vol. 36, № 1. P. 34-38.

6. Егорова Д. П., Скорняков Л. А. О структуре конгруэнций унарной алгебры // Упорядоченные множества и 
решетки. Саратов : Изд-во Сарат. ун-та, 1977. Т. 4. unary algebras. Mimeographed seminar notes. N. Y. : C. $28-40$.

U. C. Berkeley, 1967. 16 p.

7. Егорова Д. П. Структура конгруэнций унарной ал9. Карташов В. К. Независимые системы порождаюгебры // Упорядоченные множества и решетки. Саратов : Изд-во Сарат. ун-та, 1978. Т. 5. С. 11-44. щих и свойство Хопфа для унарных алгебр //Дискрет8. Johnson J., Seifert R. L. A survey of multi- $10.4213 /$ dm1027.

\title{
On Conditions for Distributivity or Modularity of Congruence Lattices of Commutative Unary Algebras
}

\begin{abstract}
V. K. Kartashov, A. V. Kartashova, V. N. Ponomarjov
Volgograd State Socio-pedagogical University, Russia, 400066, Volgograd, Lenina pr., 27, kartashovvk@yandex.ru, kartashovaan@yandex.ru

The paper is devoted to the problem of describing unary algebras whose congruence lattices have a given property. By now this problem has been solved for algebras with one unary operation. In the paper it is shown that this problem is much more difficult for arbitrary commutative unary algebras. We give some necessary conditions for such lattices to be distributive or modular. Besides, it is proved here that a lattice of all subsets of a set is isomorphic to the congruence lattice of a suitable connected commutative unary algebra.
\end{abstract}

Key words: commutative unary algebra, distributive lattice, modular lattice, congruence lattice of an algebra.

\section{References}

1. Gratzer G., Shmidt E. T. Characterizations of congruence lattices of abstract algebras. Acta Sci. Math., 1963, vol. 24, pp. 34-59.

2. Mal'tsev A. I. Algebraic Systems. Berlin, SpringerVerlag, 1976, 392 p. (Rus. ed. : Mal'tsev A. I. Algebraicheskie sistemy. Moscow, Nauka, 1970, 392 p.)

3. Skornjakov L. A. Unars. Coll. Math. Soc. J. Bolyai., 1982. vol. 29. Universal Algebra (Esztergom 1977). pp. $735-743$.

4. Skornjakov L. A. Complements in the lattice of congruences. Mathematics of the USSR-Sbornik, 1972. vol. 17, no 1. pp. 148-181. DOI: 10.1070/SM1972v017n 01ABEH001495.

5. Berman J. On the congruence lattices of unary algebras. Proc. Amer. Math. Soc., 1972. vol. 36. no 1. pp. $34-38$
6. Egorova D. P., Skornjakov L. A. O strukture kongrujencij unarnoj algebry [On congruence lattice of a unary algebra]. Uporjadochennye mnozhestva i reshetki [Ordered sets and lattices]. Saratov, Saratov Univ. Press, 1977, vol. 4. pp. 28-40 (in Russian).

7. Egorova D. P. Struktura kongrujencij unarnoj algebry [The congruence lattice of a unary algebra]. Uporjadochennye mnozhestva $i$ reshetki [Ordered sets and lattices]. Saratov, Saratov Univ. Press, 1978, vol. 5. pp. 11-44 (in Russian).

8. Johnson J., Seifert R. L. A survey of multi-unary algebras. Mimeographed seminar notes. New York, U. C. Berkeley, 1967, 16 p.

9. Kartashov V. K. Independent systems of generators and the Hopf property for unary algebras. Discrete Mathematics and Applications, 2008, vol. 18, iss. 6, pp. 625-630. DOI: 10.1515/DMA.2008.047.

УДК 512.567.5

\section{О РЕШЕТКАХ КОНГРУЭНЦИЙ ПРЯМЫХ СУММ СИЛЬНО СВЯЗНЫХ КОММУТАТИВНЫХ УНАРНЫХ АЛГЕБР}

\section{А. В. Карташова}

Кандидат фризико-математических наук, доцент кафредры алгебры, геометрии и математического анализа, Волгоградский государственный социально-педагогический университет, kartashovaan@yandex.ru

Объединение любого семейства попарно непересекающихся унарных алгебр называют их прямой суммой. Говорят, что унарная алгебра сильно связна, если она порождается любым своим элементом. В данной работе исследуется решетки конгруэнций коммутативных унарных алгебр с конечным числом операций, у которых каждая связная компонента является 